\title{
Colorimetric and Multispectral Image Acquisition Using Model-Based and Empirical Device Characterization
}

\author{
Daniel Nyström \\ Dept. of Science and Technology (ITN), Linköping University \\ SE-60174 Norrköping, Sweden \\ danny@itn.liu.se
}

\begin{abstract}
The focus of the study is high quality image acquisition in colorimetric and multispectral formats. The aim is to combine the spatial resolution of digital images with the spectral resolution of color measurement instruments, to allow for accurate colorimetric and spectral measurements in each pixel of the acquired images. An experimental image acquisition system is used, which besides trichromatic RGB filters also provides the possibility of acquiring multi-channel images, using a set of narrowband filters. To derive mappings to colorimetric and multispectral representations, two conceptually different approaches are used. In the model-based characterization, the physical model describing the image acquisition process is inverted, to reconstruct spectral reflectance from the recorded device response. In the empirical characterization, the characteristics of the individual components are ignored, and the functions are derived by relating the device response for a set of test colors to the corresponding colorimetric and spectral measurements, using linear and polynomial least squares regression. The results indicate that for trichromatic imaging, accurate colorimetric mappings can be derived by the empirical approach, using polynomial regression to CIEXYZ and CIELAB. However, accurate spectral reconstructions requires for multi-channel imaging, with the best results obtained using the model-based approach.
\end{abstract}

Keywords: Multispectral imaging, Device characterization, Spectral reconstruction, Metamerism.

\section{Introduction}

The trichromatic principle of representing color has for a long time been dominating in color imaging. The reason is the trichromatic nature of human color vision, but as the characteristics of typical color imaging devices are different from those of human eyes, there is a need to go beyond the trichromatic approach. The interest for multichannel imaging, i.e. increasing the number of color channels, has made it an active research topic with a substantial potential of application.

To achieve consistent color imaging, one needs to map the imaging-device data to the device-independent colorimetric representations CIEXYZ or CIELAB. As the color coordinates depend not only on the reflective spectrum of the object but also on 
the spectral properties of the illuminant, the colorimetric representation suffers from metamerism, i.e. objects of the same color under a specific illumination may appear different when they are illuminated by another light source. Furthermore, when the sensitivities of the imaging device differ from the CIE color matching functions, two spectra that appear different for human observers may result in identical device response. In multispectral imaging, color is represented by the object's spectral reflectance, which is illuminant independent. With multispectral imaging, different spectra are readily distinguishable, no matter they are metameric or not. The spectrum can then be transformed to any color space and be rendered under any illumination.

The focus of the paper is colorimetric and multispectral image acquisition, which requires methods for computing colorimetric and spectral data from the recorded device signals. Experiments are performed using trichromatic imaging as well as multi-channel imaging, using an experimental image acquisition system. Two conceptually different approaches for device characterization are evaluated: modelbased and empirical characterization. In the model-based approach, the physical model describing the process by which the device captures color is inverted to reconstruct spectral reflectance. In the empirical approach, the device characteristics are ignored and the mappings are derived by correlating the device response for a set of reference colors to the corresponding colorimetric and spectral measurements, using least squares regression.

\section{Model-Based Characterization}

The linear model for the image acquisition process, describing the device response to a known input, is given in Eq.1. The device response, $d_{k}$, for the $k$ :th channel is, for each pixel, given by:

$$
d_{k}=\int_{\lambda \in V} I(\lambda) F_{k}(\lambda) R(\lambda) S(\lambda) d \lambda+\varepsilon_{k}
$$

where $I(\lambda)$ is the spectral irradiance of the illumination, $F_{k}(\lambda)$ is the spectral transmittance of filter $k, R(\lambda)$ is the spectral reflectance of the object, $S(\lambda)$ is the spectral sensitivity function for the camera, $\varepsilon_{k}$ is the measurement noise for channel $k$, and $V$ is the spectral sensitivity region of the device.

The spectral characteristics of the illumination and the filters have been derived from direct measurements, using a spectroradiometer. The spectral sensitivity of the CCD camera has previously been estimated by relating the device response to the known spectral reflectance for a set of carefully selected color samples, using leastsquares regression techniques [1]. The spectral properties of the components of the image acquisition system are given in Fig. 1.

Having obtained the forward characterization function of all the components in the image acquisition system, the known spectral characteristics of the system can be 

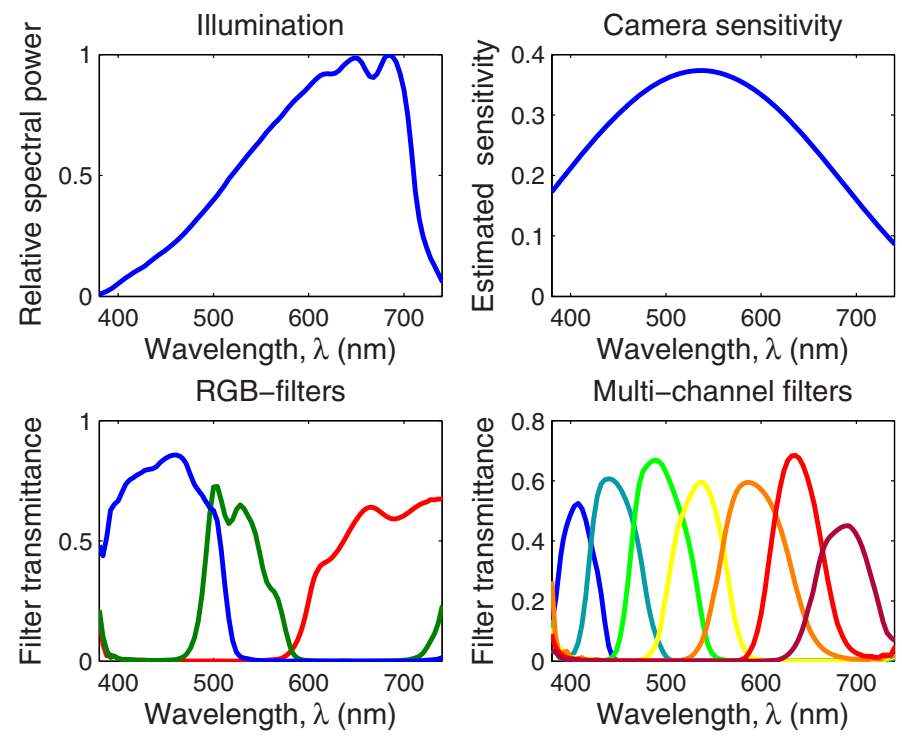

Fig. 1. Measured spectral power for the illuminant $(a)$, estimated spectral sensitivity for the camera $(b)$ and measured spectral transmittance for the RGB filters $(c)$ and the 7 multi-channel filters $(d)$

represented by a spectral transfer function [2]. The spectral transfer function, $W_{k}(\lambda)$, describes the spectral characteristics for each channel $k$, as:

$$
W_{k}(\lambda)=I(\lambda) F_{k}(\lambda) S(\lambda) .
$$

Denote the spectral signal as a discrete $N$-component vector, sampled at wavelengths $\lambda_{1}, \ldots, \lambda_{N}$, and let $\mathbf{W}$ be the $N \times K$ matrix in which each column describes the spectral transfer function of channel $k$. Then the device response vector, $\mathbf{d}$, for a sample with spectral reflectance $\mathbf{r}$ is given by:

$$
\mathbf{d}=\mathbf{W}^{t} \mathbf{r} .
$$

When inverting the model, we seek the $N \times K$ reconstruction matrix $\mathbf{M}$ that reconstructs the spectral reflectance, $\check{\mathbf{r}}$, from the camera response $\mathbf{d}$, as:

$$
\breve{\mathbf{r}}=\mathbf{M d} .
$$

The most straightforward approach to derive the reconstruction matrix is to simply invert Eq. 3, using the pseudo-inverse approach, giving the reconstruction operator:

$$
\mathbf{M}_{0}=\left(\mathbf{W} \mathbf{W}^{t}\right)^{-1} \mathbf{W}=\left(\mathbf{W}^{t}\right)^{-} \text {. }
$$


where $\left(\mathbf{W}^{\mathrm{t}}\right)^{-}$denotes for the More-Penrose pseudo-inverse of $\mathbf{W}^{\mathrm{t}}$. Generally, the pseudo-inverse reconstruction is sensitive to noise, which makes the approach not always useful in practices. When $K<N$, i.e. the number of color channels $K$ is less than the number of spectral sampling points $N$, the matrix $\mathbf{W}$ is of insufficient rank and the algebraic equations are underdetermined. Further more, this method minimizes the Euclidian distance in the camera response domain (i.e. between $\mathbf{d}$ and $\mathbf{W}^{\mathrm{t}} \breve{\mathbf{r}}$ ), which does not necessarily mean that the reconstructed spectrum will be close to the real spectrum [3].

Another approach is to instead seek another reconstruction matrix, $\mathbf{M}_{1}$, which minimizes the Euclidian distance between the reconstructed spectrum and the original spectrum [3]. By exploiting the a priori information that the vast majority of reflectance spectra for real and man-made surfaces are smooth functions of wavelength ([4], [5]), it can be assumed that the spectrum can be represented by a linear combination of a set of smooth basis functions, $\mathbf{B}=\left[\mathbf{b}_{1}, \mathbf{b}_{2} \ldots \mathbf{b}_{\mathrm{p}}\right]$. This gives the reconstruction operator $\mathbf{M}_{1}$, which minimizes the RMS spectral difference of the reconstructed spectrum, as (refer to [3], for details):

$$
\mathbf{M}_{1}=\mathbf{B B}^{t} \mathbf{W}\left(\mathbf{W}^{t} \mathbf{B} \mathbf{B}^{t} \mathbf{W}\right)^{-1} \text {. }
$$

The base functions, B, can consist of a set of real, measured spectral reflectances, which then should be representative to the reflectance of samples that is likely to be encountered in the image acquisition system. An alternative to spectral basis is to simply let $\mathbf{B}$ consist of a set of Fourier basis functions.

\section{Empirical Characterization}

In empirical characterization, colorimetric and spectral data are derived using a "black box" approach, i.e. without explicitly modeling the device characteristics. By correlating the device response for a training set of color samples to the corresponding colorimetric or spectral values, the characterization functions are derived using least squares regression.

The characterization functions derived using empirical approaches will be optimized only for a specific set of conditions, including the illuminant, the media and the colorant. Once the conditions change, e.g. a different substrate or a different print mechanism, the characterization has to be re-derived in order to obtain good accuracy (see for example [6], [7]). The dependency on the illuminant is not an issue when the light source is fixed and can be considered as a property of the system. However, the fact that the characterization function is also media- and colorant dependent is a major drawback, preventing the characterization function from being applied to arbitrary combinations of media and colorants.

\subsection{Spectral Regression}

Even though empirical approaches are mainly used to derive mappings to colorimetric data, CIEXYZ or CIELAB, there have been attempts to reconstruct spectral reflectance [8]. The spectral reconstruction matrix, $\mathbf{M}$, is now derived entirely based 
on the recorded device response to a set of training samples, i.e. ignoring the spectral characteristics of the imaging system. If the spectral reflectance for a set of $T$ training samples are collected into a $T \times N$ matrix $\mathbf{R}=\left[\mathbf{r}_{1}, \ldots, \mathbf{r}_{T}\right]$ and the corresponding device responses into a $T \times K$ matrix $\mathbf{D}=\left[\mathbf{d}_{1}, \ldots, \mathbf{d}_{\mathrm{T}}\right]$, then the linear relationship is given by:

$$
\mathbf{R}=\mathbf{D M} \text {. }
$$

and the optimal $K \times N$ spectral reconstruction matrix $\mathbf{M}$ is then given by:

$$
\mathbf{M}=\left(\mathbf{D}^{t} \mathbf{D}\right)^{-1} \mathbf{D}^{t} \mathbf{R}=(\mathbf{D})^{-} \mathbf{R} .
$$

In the same way as for the model-based approach, the reconstructed spectra can be represented as linear combinations of a set of basis functions.

\subsection{Colorimetric Regression}

A common approach to derive colorimetric data is to use polynomial regression from device values to CIEXYZ [9]. For example, the inverse characterization function of a 3-channel system, mapping RGB values to XYZ tristimulus values, is obtained by expressing $\mathrm{XYZ}$ as polynomial functions of $\mathrm{R}, \mathrm{G}$ and $\mathrm{B}$. As an example, a second order polynomial approximation is given by:

$$
[X Y Z]=\left[1, R, G, B, R^{2}, R G, R B, G^{2}, G B, B^{2}\right]\left[\begin{array}{ccc}
w_{X, 1} & w_{Y, 1} & w_{Z, 1} \\
w_{X, 2} & w_{Y, 2} & w_{Z, 2} \\
& \ldots & \\
w_{X, 10} & w_{Y, 10} & w_{Z, 10}
\end{array}\right] .
$$

or, generally:

$$
\mathbf{c}=\mathbf{p A} .
$$

where $\mathbf{c}$ is the colorimetric output vector, $\mathbf{p}$ is the $Q$-component vector of polynomial terms derived from the device data $\mathbf{d}$, and $\mathbf{A}$ is the $Q \times n$ matrix of polynomial weights. The optimal matrix of polynomial weights, $\mathbf{A}$, is then given by:

$$
\mathbf{A}=\left(\mathbf{P}^{t} \mathbf{P}\right)^{-1} \mathbf{P}^{t} \mathbf{C}=(\mathbf{P})^{-} \mathbf{C} .
$$

The drawback with using regression to CIEXYZ is that the RMS error in XYZ color space, which is minimized in the regression, is not closely related to the perceived color difference. If the final aim is to derive data in CIELAB color space, it is therefore preferable to use regression directly in the CIELAB domain, i.e. to minimize the CIE 1976 color difference $\Delta \mathrm{E}_{\mathrm{ab}}$, which provides a better correspondence to the visual color difference [3]. Since the relationship between device data and CIELAB is not linear, a non-linear pre-processing step of the device values using a cubic root function has been proposed, i.e. using $R^{1 / 3}, G^{1 / 3}, B^{1 / 3}$ in the regression [3]. The cubic root function originates from the CIELAB transformation, which involves a cubic root function of the $\mathrm{XYZ}$ tristimulus values. 


\section{The Image Acquisition System}

The images are captured using a monochrome CCD camera with 12 bit dynamic range, specially designed for scientific imaging. The illumination is provided using a tungsten halogen lamp through optical fibers, which offers an adjustable angle of incidence, as well as the possibility of using a backlight setup. Color images are sequentially captured, using filters mounted in a filter wheel in front of the light source. By using this color sequential method, there is no need for any interpolation or de-mosaicing scheme, as is the case for conventional digital cameras. Besides the trichromatic RGB-filters, the filter wheel also contains a set of 7 interference filters, allowing for the acquisition of multi-channel images. The interference filters have been selected to cover the visible spectrum with equally spaced pass bands, see Fig. 1 .

Since the accuracy of the characterization will always be limited by the stability and uniformity of a given device, the characterization procedure has been preceded by a thorough calibration of the system. All the components have been controlled with respect to linearity, temporal stability and spatial uniformity.

\section{Experimental Setup}

The evaluation of the spectral and colorimetric reconstructions requires for the acquisition of spectral and colorimetric data for a set of test colors, along with the corresponding device response. Spectral measurements of the color-patches are performed using a spectroradiometer, placed in the same optical axis as the CCDcamera, using the $45^{\circ} / 0^{\circ}$ measurement geometry. For each color patch, the mean reflectance spectrum from 5 sequential measurements is computed. The colorimetric data have been computed using standard formulae under the D65 standard illuminant. Correspondingly, the camera response values have been acquired under identical conditions. Before the mean values are computed, the images are corrected for dark current and $\mathrm{CCD}$ gain.

For reference colors to evaluate the results of the model-based spectral reconstruction, 25 color patches from NCS are used. For the empirical characterization, a training set of 50 printed test colors are used to derive the characterization functions. For the evaluation, 50 independent colors are used, printed using the same substrate and conditions as the training set. Since characterization functions derived by least squares regression will always be optimized for the specific training set, it is important to use an independent set of evaluation colors to guard against a model that overfits the training set, giving unrealistically good results [10].

As basis functions we evaluate spectral basis, using a database of real spectra available from NCS, as well as Fourier basis. Five basis functions are used, corresponding to the first five Fourier basis functions and to the five singular vectors corresponding to the most significant singular values in the spectral autocorrelation function of the spectral database, using the principle eigenvector method [3]. 


\section{Experimental Results}

\subsection{Spectral Reconstruction}

Spectral data has been reconstructed from the recorded device response, using the pseudo-inverse (PI) method, as well as using spectral and Fourier basis, for the model-based and empirical approaches, respectively. The results are evaluated using the spectral RMS error, corresponding to the Euclidian distance in spectral reflectance space, between the original and the reconstructed spectra. The CIE 1976 color difference $\Delta \mathrm{E}_{\mathrm{ab}}$ is also computed, to provide a measure of the perceived color difference between the spectra.

Table 1 lists the mean and maximum reconstruction errors, for the different characterization methods. Examples of reconstructed spectra, compared to the corresponding measured spectra, are displayed in Fig 2.

The results show that for the model-based approach, trichromatic imaging is not sufficient to achieve spectral or colorimetric accuracy. For the multi-channel images, the results improve dramatically. Spectral basis and Fourier basis lead to equivalent results in terms of the RMS difference, while the colorimetric results are in favor of the spectral basis. The pseudo-inverse solution is somewhat noisy and suffers from larger RMS difference. However, the general shapes of the reconstructed spectra follow the real spectra well, resulting small colorimetric errors. Clearly, the PImethod produces spectral reconstructions that are close to metameric matches.

For the empirical characterization using trichromatic imaging, the pseudo-inverse method is superior to the corresponding model-based results. However, the improvement when applying the different basis functions is not as evident, and the best results for the model-based approach could not be achieved. The results using multi-channel imaging is comparable to the corresponding model-based approach in terms of spectral RMS difference, but produces larger colorimetric errors.

Table 1. Spectral reconstruction errors, in terms of spectral RMS error and $\Delta \mathrm{E}_{\mathrm{ab}}$

\begin{tabular}{|c|c|c|c|c|c|c|}
\hline & \multirow[b]{2}{*}{ Data } & \multirow[b]{2}{*}{ Method } & \multicolumn{2}{|c|}{ RMS } & \multicolumn{2}{|c|}{$\Delta \mathbf{E}_{a b}$} \\
\hline & & & $\operatorname{Max}$ & Mean & $\operatorname{Max}$ & Mean \\
\hline \multirow{6}{*}{ 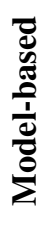 } & RGB & PI & 0.0706 & 0.0230 & 24.35 & 14.80 \\
\hline & & Spectral & 0.0041 & 0.0014 & 15.87 & 4.170 \\
\hline & & Fourier & 0.0155 & 0.0049 & 18.75 & 8.287 \\
\hline & Multi & PI & 0.0092 & 0.0030 & 4.364 & 1.529 \\
\hline & & Spectral & 0.0039 & 0.0012 & 4.218 & 1.816 \\
\hline & & Fourier & 0.0040 & 0.0011 & 7.271 & 2.112 \\
\hline \multirow{6}{*}{ 氕 } & RGB & PI & 0.0082 & 0.0023 & 13.22 & 7.532 \\
\hline & & Spectral & 0.0062 & 0.0031 & 12.49 & 7.444 \\
\hline & & Fourier & 0.0072 & 0.0035 & 13.81 & 6.897 \\
\hline & Multi & PI & 0.0030 & 0.0004 & 6.899 & 3.908 \\
\hline & & Spectral & 0.0040 & 0.0018 & 9.320 & 5.525 \\
\hline & & Fourier & 0.0052 & 0.0023 & 13.90 & 6.085 \\
\hline
\end{tabular}



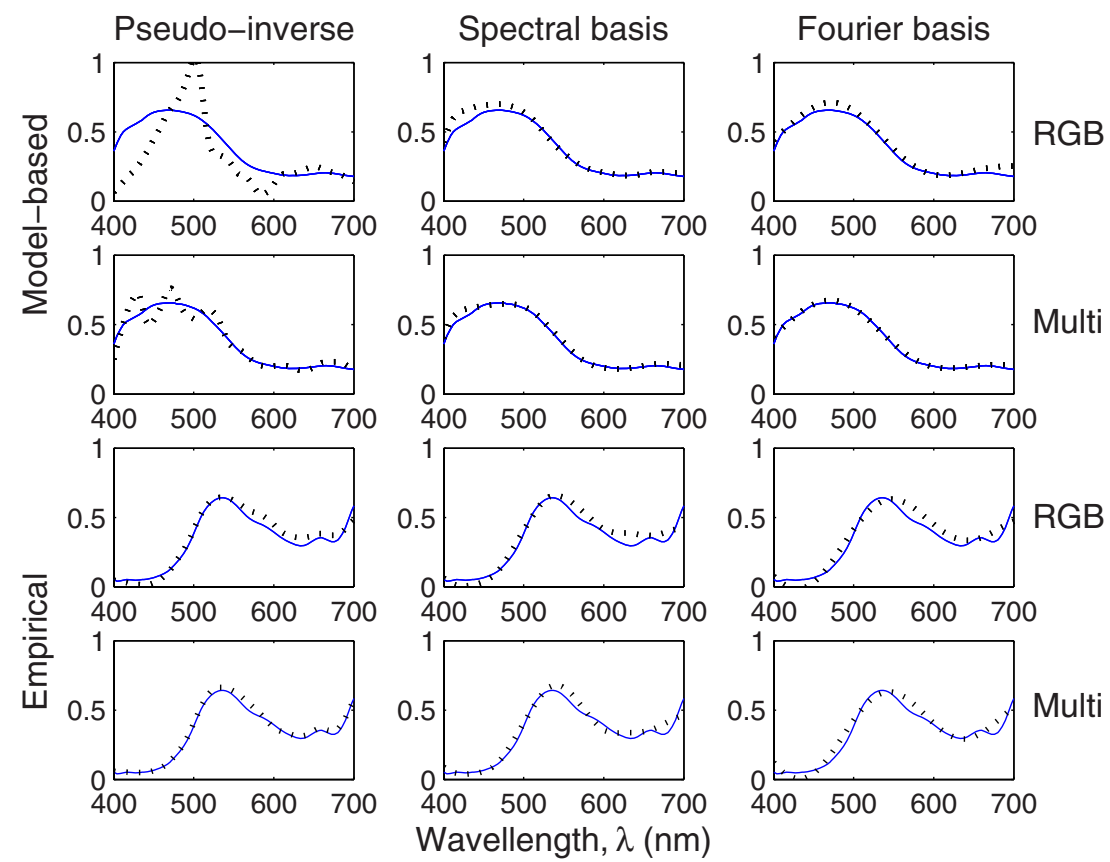

Fig. 2. Reconstructed spectral reflectance (dashed lines) compared to measured (full lines). Results for the model-based characterization using RGB (first row) and multi-channel imaging (second row), and the empirical characterization using RGB (third row) and multi-channel imaging (last row).

\subsection{Colorimetric Regression}

For the polynomial regression, there are numerous ways to build the approximation functions, $\mathbf{p}$, and the number of terms, $Q$, increases rapidly for higher order polynomials. Among the different polynomials evaluated [11], the polynomials found to give the best results, for RGB and multi-channel imaging, respectively, were:

$$
\begin{aligned}
\mathbf{p}_{R G B}= & {\left[1, R, G, B, R^{2}, R G, R B, G^{2}, G B, B^{2}, R^{3}, R^{2} G, R^{2} B, R G^{2},\right.} \\
& \left.R G B, R B^{2}, G^{3}, G^{2} B, G B^{2}, B^{3}\right] \\
\mathbf{p}_{\text {multi }}= & {\left[1, M_{1}, M_{2}, M_{3}, M_{4}, M_{5}, M_{6}, M_{7}, M_{1}{ }^{2}, M_{2}{ }^{2}, M_{3}{ }^{2}, M_{4}{ }^{2},\right.} \\
& \left.M_{5}{ }^{2}, M_{6}{ }^{2}, M_{7}{ }^{2}, M_{1} M_{2} M_{3} M_{4} M_{5} M_{6} M_{7}\right]
\end{aligned}
$$

Table 2 lists the results for the colorimetric regression to CIEXYZ and CIELAB, using the polynomials according to Eqs. $12 \& 13$. For the regression directly to CIELAB, the non-linear pre-processing step has been used, which proved to be superior to regression to CIELAB using the unprocessed device response. To investigate the media-dependency, cross-media characterization was carried out, 
using the characterization functions derived for the printed training set to reconstruct colorimetric data for the NCS color patches.

The results show that colorimetric regression directly to CIEXYZ and CIELAB gives a good colorimetric accuracy. Noticeable is that the results from the trichromatic RGB images are comparable to the multi-channel results. However, for cross-media characterization, the reconstruction errors increase dramatically, illustrating the strong media dependency of the method.

Table 2. The results for the colorimetric regression to CIEXYZ and CIELAB

\begin{tabular}{|c|c|c|c|c|c|c|}
\hline & \multirow[b]{2}{*}{ Data } & \multirow[b]{2}{*}{ Regression } & \multicolumn{2}{|c|}{$\Delta \mathbf{X Y Z}$} & \multicolumn{2}{|c|}{$\Delta \mathbf{E}_{\mathrm{ab}}$} \\
\hline & & & $\operatorname{Max}$ & Mean & $\operatorname{Max}$ & Mean \\
\hline & RGB & CIEXYZ & 3.453 & 0.904 & 4.558 & 2.086 \\
\hline & & CIELAB & & & 4.317 & 1.722 \\
\hline & Multi & CIEXYZ & 3.240 & 0.945 & 3.765 & 1.942 \\
\hline & & CIELAB & & & 3.846 & 1.957 \\
\hline \multirow{4}{*}{ 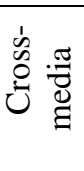 } & RGB & CIEXYZ & 25.55 & 12.96 & 16.67 & 9.494 \\
\hline & & CIELAB & & & 18.29 & 8.417 \\
\hline & Multi & CIEXYZ & 13.71 & 5.366 & 26.00 & 9.781 \\
\hline & & CIELAB & & & 18.76 & 8.877 \\
\hline
\end{tabular}

\section{Summary}

The focus of this study has been colorimetric and multispectral image acquisition, using both trichromatic and multi-channel imaging. To reconstruct colorimetric and spectral data from the recorded device response, two conceptually different approaches have been investigated: model-based and empirical characterization. In the model-based approach, the spectral model of the image acquisition system is inverted. A priori knowledge on the smooth nature of spectral reflectance was utilized by representing the reconstructed spectra as linear combinations of basis functions, using Fourier basis and a set of real reflectance spectra. In the empirical approach, the spectral characteristics of the system are ignored and the mappings are derived by relating the recorded device response to colorimetric and spectral data for a set of training colors, using least squares regression techniques.

The results have showed that when only trichromatic imaging is available, the best method for colorimetric imaging is the empirical approach, using polynomial regression. However, because of the media-dependency, this requires for the characterization functions to be derived for each combination of media and colorants. For multispectral imaging, reconstructing the spectral reflectance of objects, multichannel images are required to obtain the highest accuracy. The best results were obtained with the model-based approach, using multi-channel images combined with spectral basis. The model-based approach provides the additional advantage of being general, since it is derived based on the spectral characteristics of the image acquisition system, rather than on the characteristics of a set of color samples. However, the model-based approach requires for multi-channel imaging to obtain a satisfactory spectral or colorimetric accuracy. 


\section{References}

1. Nyström, D., Kruse, B.: Colorimetric Device Characterization for Accurate Color Image Acquisition. In: Advances in Printing and Media Technology, vol. 33 (2006)

2. Farell, J.E., et al.: Estimating Spectral Reflectances of Digital Artwork. In: Proc. Chiba Conference of Multispectral Imaging (1999)

3. Hardeberg, J.Y.: Acquisition and Reproduction of Color Images: Colorimetric and Multispectral Approaches, Dissertation.com, ISBN 1-58112-135-0 (2001)

4. Maloney, L.T.: Evaluation of linear models of surface spectral reflectance with small numbers of parameters. In: J. Opt. Soc. Am. A, 3 (1986)

5. Connah, D. et al.: Recovering spectral information using digital camera systems. Coloration technology 117, 309-312 (2001)

6. Pan, Z., et al.: Color Scanner Characterization with Scan Targets of Different Media Types and Printing Mechanisms. In: Proc. Color Imaging: Device-Independent Color, Color Hardcopy, and Graphic Arts VI, pp. 58-63 (2001)

7. Andersson, M., et al.: The Substrate influence on color measurement, In: Proc. IS\&T NIP 19, New Orleans (2003)

8. Solli, M., et al.: Color Measurements with a Consumer Digital Camera Using Spectral Estimation Techniques. In: Proc. Scandinavian Conference on Image Analysis, pp. 105114 (2005)

9. Hong, G., Lou, M.R.: A Study of Digital Camera Colorimetric Characterization Based on Polynomial Modeling. In: COLOR research and application, vol. 26(1) (2001)

10. Cheung, V. et al.: Characterization of trichromatic color cameras by using a new multispectral imaging technique. J. Opt. Soc. Am. A, 22(7), 1231-1240 (2005)

11. Nyström, D.: Colorimetric and Multispectral Image Acquisition. Licentiate Thesis No. 1289, Linköping University, Sweden (2006) 